\title{
Nonstationary CARS by Polaritons
}

\author{
Vladimir Feshchenko', Galina Feshchenko² \\ ${ }^{1}$ Dawson College, Montreal, Canada \\ ${ }^{2}$ Vanier College, Montreal, Canada \\ Email: vfeshchenko@dawsoncollege.qc.ca, feshcheg@vaniercollege.qc.ca
}

How to cite this paper: Feshchenko, V. and Feshchenko, G. (2020) Nonstationary CARS by Polaritons. Journal of Applied Mathematics and Physics, 8, 1949-1958. https://doi.org/10.4236/jamp.2020.89146

Received: August 3, 2020

Accepted: September 24, 2020

Published: September 27, 2020

Copyright $\odot 2020$ by author(s) and Scientific Research Publishing Inc. This work is licensed under the Creative Commons Attribution International License (CC BY 4.0).

http://creativecommons.org/licenses/by/4.0/

\section{(c) (i) Open Access}

\begin{abstract}
One of the significant problems of molecular spectroscopy is the determination and detailed analysis of how molecular vibrations are dephased. The dephasing of infrared-active (IR-active) vibrations of molecules was investigated by IR absorption spectroscopy. Pulse methods were used to investigate IR-vibrations as well. These methods revealed such coherent nonstationary effects as optical nutation, damping of the free polarization, photon echo, etc. New means of studying dephasing processes were uncovered by the method of nonstationary (time-domain) coherent anti-Stokes Raman scattering (CARS) spectroscopy. However, there are some aspects of CARS that still are not fully covered. One of them is related to Raman scattering by polaritons in dipole-active crystals whereas the second one is the increase of efficiency of CARS (minimization of the wave mismatch, the relationship between pulse width and the relaxation time, etc.). The purpose of the present research to study the case of "extreme" coherency between all interacting pulses (the duration of each pulse is smaller than characteristic times and those pulses are traveling with the same speed) in dipole-active crystals. In this research, we analyzed the process of simultaneous propagation of three waves (anti-Stokes, Stokes, and the pump) under CARS by polaritons. We have found some solutions modeling such simultaneous propagation. We also found the expression for the gain factor for such scattering. The gain factor was evaluated under the assumption of a given stationary pump field. It was shown that the typical values of the relative intensities were consistent with the experimental results.
\end{abstract}

\section{Keywords}

Coherent Anti-Stokes Raman Scattering, Spectroscopy, Polaritons, Stimulated Raman Scattering

\section{Introduction}

Maker and Terhune were first who demonstrated the CARS technique [1]. In [1] 
was also shown that the efficiency of the CARS generation is the function of the third-order susceptibilities which were extensively studied in the experiments of the nonlinear properties of solids and liquids [2] [3] [4]. Since then CARS spectroscopy has become a powerful technique in many fields of knowledge such as physics, biology, chemistry, healthcare, etc. [5] [6] [7] [8] [9]. The CARS imaging proved its efficiency in cancer diagnosis as well [10] [11]. Begley et al. were among the researches who summarized the important advantages of vibrational spectroscopy based on nonlinear anti-Stokes generation [12] [13] [14]. This technique went to the next level when the ultrashort laser pulses resulted in the possibility of the coherent excitation of multiple Raman modes [15] [16] [17]. For example, the method of nonstationary (time-domain) CARS spectroscopy permitted direct observation of vibration dephasing in an ensemble of atoms or molecules or even in the simplest system-molecular hydrogen [18]. The further increase in efficiency would result from simultaneous propagation in the medium of all interacting waves. In [19] [20] [21] we considered the cases of Raman scattering by polaritons in dipole-active crystals. In this paper, we considered the theoretical modeling of the processes of nonstationary CARS by polaritons in dipole-active crystals.

\section{Basic Principles and Equations}

In this paper, we consider the nonlinear interaction of four electromagnetic waves: anti-Stokes, Stokes, pump (laser), and polariton. Those waves are assumed to be linearly polarized plane waves. It is also assumed that the nonlinear medium takes a form of a layer bounded by the planes $z=0$ and $z=L$. The pump wave

$$
\vec{E}_{l}(\vec{r}, t)=\hat{e}_{l} A_{l}(z, t) \exp \left[i\left(k_{l}^{z} z-\omega_{l} t\right)\right]+\text { c.c. }
$$

propagates along the z-axis. The subscripts $a, l, s$, and $p$ denote the anti-Stokes, pump (laser), Stokes, and polariton wave fields, $\omega_{a, l, s, p}$ are the frequencies, $n_{a, l, s, p}$ and $\vec{k}_{a, l, s, p}$ are the refractive indices and the wave vectors in the unpumped medium, and $\hat{e}_{a, l, s, p}$ are the real unit vectors of electromagnetic fields. The nonlinear medium is assumed to be nonmagnetic and transparent at the frequencies $\omega_{a, l, s}$. We use the anti-Stokes, Stokes, and polariton fields in the form

$$
\begin{aligned}
\vec{E}_{a}(\vec{r}, t) & =\hat{e}_{a} A_{a}(z, t) \exp \left[i\left(k_{a}^{z} z-\omega_{a} t\right)\right]+c . c ., \\
\vec{E}_{s}(\vec{r}, t) & =\hat{e}_{s} A_{s}(z, t) \exp \left[i\left(k_{s}^{z} z-\omega_{s} t\right)\right]+c . c ., \\
\vec{E}_{p}(\vec{r}, t) & =\hat{e}_{p} A_{p}(z, t) \exp \left[i\left(W^{z} z-\omega_{p} t\right)\right]+c . c .,
\end{aligned}
$$

where $k_{a, s}=q_{a, s} n_{a, s} ; \quad q_{a, s}=\omega_{a, s} / c ; W^{z}=k_{l}^{z}-k_{s}^{z} ; \omega_{p}=\omega_{l}-\omega_{s}$.

In the process of CARS, the nonlinear interaction of two electromagnetic waves $\omega_{l, s}$ results in the generation of anti-Stokes and polariton waves. The system of shortened equations for the amplitudes $A_{a, l, s, p}$ is obtained from Max- 
well's equations by using the standard approximation of slowly-varying amplitudes [22] and takes the form

$$
\begin{gathered}
\frac{\partial A_{a}}{\partial z}+\frac{1}{v_{a}^{z}} \frac{\partial A_{a}}{\partial t}=i \frac{2 \pi \omega_{a}}{c n_{a} \cos \left(\theta_{a}^{z}\right)}\left\{\chi_{a} A_{l} A_{p} \mathrm{e}^{i \Delta k^{z} z}+\gamma_{a}\left(\left|A_{l}\right|^{2}+\left|A_{s}\right|^{2}\right) A_{a}\right\}, \\
\frac{\partial A_{l}}{\partial z}+\frac{1}{v_{l}^{z}} \frac{\partial A_{l}}{\partial t}=i \frac{2 \pi \omega_{l}}{c n_{l} \cos \left(\theta_{l}^{z}\right)}\left\{\chi_{l 1} A_{s} A_{p}+\chi_{l 2} A_{a} A_{p}^{*} \mathrm{e}^{-i \Delta k^{z} z}\right\}, \\
\frac{\partial A_{s}}{\partial z}+\frac{1}{v_{s}^{z}} \frac{\partial A_{s}}{\partial t}=i \frac{2 \pi \omega_{s}}{c n_{s} \cos \left(\theta_{s}^{z}\right)}\left\{\chi_{s} A_{l} A_{p}^{*}+\gamma_{s}\left(\left|A_{l}\right|^{2}+\left|A_{s}\right|^{2}\right) A_{s}\right\}, \\
\frac{\partial A_{p}^{*}}{\partial z}+\frac{1}{v_{p}^{z}} \frac{\partial A_{p}^{*}}{\partial t}=i \frac{q_{p}^{2} \varepsilon_{p}^{\infty}}{2 W^{z}}\left(\frac{W^{2}}{q_{p}^{2} \varepsilon_{p}^{\infty}}-1\right) A_{p}^{*}-i \frac{2 \pi q_{p}^{2}}{W^{z}}\left\{\chi_{p 1} A_{l}^{*} A_{s}+\chi_{p 2} A_{a}^{*} A_{1} \mathrm{e}^{i \Delta k^{z} z}\right\},
\end{gathered}
$$

where $\chi_{a}, \chi_{11,12}, \chi_{s}, \chi_{p 1, p 2}, \gamma_{a, s}$ are the corresponding tensor contractions of non-resonance quadratic and cubic nonlinear polarizabilities with unit vectors of polarization of interacting waves; $e_{p}^{\infty}$ is the non-resonance part of dielectric permeability at frequency $\omega_{p} ; v_{a, l, s, p}^{z}$ are $z$-components of velocities of waves on $\omega_{a, l, s, p} ; \Delta k^{z} \equiv k_{l}^{z}+W^{z}-k_{a}^{z}$ is the wave mismatch between the pump, polariton, and anti-Stokes waves.

Given the strong polariton absorption we have [23]

$$
\left|\frac{\partial A_{p}^{*}}{\partial z}\right| \approx\left|\frac{1}{v_{p}^{z}} \frac{\partial A_{p}^{*}}{\partial t}\right| \ll \frac{q_{p}^{2} \varepsilon_{p}^{\infty}}{2 W^{z}}\left(\frac{W^{2}}{q_{p}^{2} \varepsilon_{p}^{\infty}}-1\right) A_{p}^{*},
$$

so that we can neglect in (8) the terms with the derivatives after which this equation yields

$$
A_{p}^{*}=\frac{4 \pi q_{p}^{2}}{\left(W^{2}-q_{p}^{2} \varepsilon_{p}^{\infty}\right)}\left(\chi_{p 1} A_{1}^{*} A_{s}+\chi_{p 2} A_{a}^{*} A_{1} \mathrm{e}^{i \Delta k^{2} z}\right) .
$$

If we insert the obtained expression for the amplitude of polariton wave in (5)-(6), we get a system of 3 differential equations for $A_{a, l, s}$ as follows:

$$
\begin{aligned}
& \frac{\partial A_{a}}{\partial z}+\frac{1}{v_{a}^{z}} \frac{\partial A_{a}}{\partial t} \\
& =i \frac{2 \pi \omega_{a}}{c n_{a} \cos \left(\theta_{a}^{2}\right)}\left\{\frac{4 \pi q_{p}^{2} \chi_{a} \chi_{p 1}^{*}}{\left(W^{2}-q_{p}^{2} \varepsilon_{p}^{\infty}\right)} A_{l}^{2} A_{s}^{*} \mathrm{e}^{i \Delta k^{2} z}+\gamma_{a 1}\left|A_{1}\right|^{2} A_{a}+\gamma_{a}\left|A_{s}\right|^{2} A_{a}\right\}, \\
& \frac{\partial A_{1}}{\partial z}+\frac{1}{v_{l}^{z}} \frac{\partial A_{1}}{\partial t}=i \frac{2 \pi \omega_{l}}{c n_{l} \cos \left(\theta_{l}^{z}\right)}\left\{\frac{4 \pi q_{p}^{2}\left(\chi_{11} \chi_{p 2}^{*}+\chi_{12} \chi_{p 1}\right)}{\left(W^{2}-q_{p}^{2} \varepsilon_{p}^{\infty}\right)} A_{a} A_{l}^{*} A_{s} \mathrm{e}^{-i \Delta k^{z_{z}} z}\right. \\
& \left.+\frac{4 \pi q_{p}^{2} \chi_{12} \chi_{p 2}}{\left(W^{2}-q_{p}^{2} \varepsilon_{p}^{\infty}\right)}\left|A_{a}\right|^{2} A_{1}+\gamma_{l 1}\left|A_{s}\right|^{2} A_{1}+\gamma_{l}\left|A_{1}\right|^{2} A_{l}\right\} \text {, } \\
& \frac{\partial A_{s}}{\partial z}+\frac{1}{v_{s}^{z}} \frac{\partial A_{s}}{\partial t} \\
& =i \frac{2 \pi \omega_{s}}{c n_{s} \cos \left(\theta_{s}^{z}\right)}\left\{\frac{4 \pi q_{p}^{2} \chi_{s} \chi_{p 2}}{\left(W^{2}-q_{p}^{2} \varepsilon_{p}^{\infty}\right)} A_{l}^{2} A_{a}^{*} \mathrm{e}^{i \Delta k^{2} z}+\gamma_{s 1}\left|A_{1}\right|^{2} A_{s}+\gamma_{s}\left|A_{s}\right|^{2} A_{s}\right\},
\end{aligned}
$$


where $\quad q_{p} \equiv \omega_{p} / c, \quad \gamma_{a 1} \equiv \gamma_{a}+\frac{4 \pi q_{p}^{2} \chi_{a} \chi_{p 2}^{*}}{\left(W^{2}-q_{p}^{2} \varepsilon_{p}^{\infty}\right)}, \quad \gamma_{s 1} \equiv \gamma_{s}+\frac{4 \pi q_{p}^{2} \chi_{s} \chi_{p 1}}{\left(W^{2}-q_{p}^{2} \varepsilon_{p}^{\infty}\right)}, \quad$ and $\gamma_{l 1} \equiv \gamma_{l}+\frac{4 \pi q_{p}^{2} \chi_{l 1} \chi_{p 1}^{*}}{\left(W^{2}-q_{p}^{2} \varepsilon_{p}^{\infty}\right)}$.

The system (11)-(13) can be simplified if we use new variables

$$
\begin{aligned}
A_{a}^{\prime} & \equiv A_{a} \mathrm{e}^{-\frac{i \Delta k^{2} z}{2}} \\
\text { and } A_{s}^{\prime} & \equiv A_{s} \mathrm{e}^{-\frac{i \Delta k^{2} z}{2}} .
\end{aligned}
$$

The system (11)-(13) in terms of $A_{a, s}^{\prime}$ can be written as follows:

$$
\begin{aligned}
& \frac{\partial A_{a}^{\prime}}{\partial z}+\frac{1}{v_{a}^{z}} \frac{\partial A_{a}^{\prime}}{\partial t}+\frac{i \Delta k^{z}}{2} A_{a}^{\prime} \\
& =i \frac{2 \pi \omega_{a}}{c n_{a} \cos \left(\theta_{a}^{z}\right)}\left\{\frac{4 \pi q_{p}^{2} \chi_{a} \chi_{p 1}^{*}}{\left(W^{2}-q_{p}^{2} \varepsilon_{p}^{\infty}\right)} A_{l}^{2} A_{s}^{\prime^{*}}+\gamma_{a 1}\left|A_{1}\right|^{2} A_{a}^{\prime}+\gamma_{a}\left|A_{s}\right|^{2} A_{a}^{\prime}\right\}, \\
& \frac{\partial A_{l}}{\partial z}+\frac{1}{v_{l}^{2}} \frac{\partial A_{l}}{\partial t}=i \frac{2 \pi \omega_{l}}{c n_{l} \cos \left(\theta_{l}^{2}\right)}\left\{\frac{4 \pi q_{p}^{2}\left(\chi_{11} \chi_{p 2}^{*}+\chi_{12} \chi_{p 1}\right)}{\left(W^{2}-q_{p}^{2} \varepsilon_{p}^{\infty}\right)} A_{a}^{\prime} A_{s}^{\prime} A_{l}^{*}\right. \\
& \left.\quad+\gamma_{l 1}\left|A_{s}^{\prime}\right|^{2} A_{1}+\gamma_{l}\left|A_{l}\right|^{2} A_{1}+\frac{4 \pi q_{p}^{2} \chi_{12} \chi_{p 2}}{\left(W^{2}-q_{p}^{2} \varepsilon_{p}^{\infty}\right)}\left|A_{a}^{\prime}\right|^{2} A_{l}\right\}, \\
& \frac{\partial A_{s}^{\prime}}{\partial z}+\frac{1}{v_{s}^{z}} \frac{\partial A_{s}^{\prime}}{\partial t}+\frac{i \Delta k^{z}}{2} A_{s}^{\prime} \\
& =i \frac{2 \pi \omega_{s}}{c n_{s} \cos \left(\theta_{s}^{z}\right)}\left\{\frac{4 \pi q_{p}^{2} \chi_{s} \chi_{p 2}}{\left(W^{2}-q_{p}^{2} \varepsilon_{p}^{\infty}\right)} A_{l}^{2} A_{a}^{\prime *}+\gamma_{s 1}\left|A_{1}\right|^{2} A_{s}^{\prime}+\gamma_{s}\left|A_{s}^{\prime}\right|^{2} A_{s}^{\prime}\right\} .
\end{aligned}
$$

And, finally, if we assume a "week" wave mismatch at Stokes and anti-Stokes frequencies, that is

$$
\left|\frac{\partial A_{a, s}^{\prime}}{\partial z}+\frac{1}{v_{a, s}^{z}} \frac{\partial A_{a, s}^{\prime}}{\partial t}\right| \gg \frac{\Delta k^{z}}{2} A_{a, s}^{\prime},
$$

then the final system of equations simulating CARS can be expressed as

$$
\begin{aligned}
& \frac{\partial A_{a}^{\prime}}{\partial z}+\frac{1}{v_{a}^{z}} \frac{\partial A_{a}^{\prime}}{\partial t} \\
& =i \frac{2 \pi \omega_{a}}{c n_{a} \cos \left(\theta_{a}^{2}\right)}\left\{\frac{4 \pi q_{p}^{2} \chi_{a} \chi_{p 1}^{*}}{\left(W^{2}-q_{p}^{2} \varepsilon_{p}^{\infty}\right)} A_{1}^{2} A_{s}^{\prime *}+\gamma_{a 1}\left|A_{1}\right|^{2} A_{a}^{\prime}+\gamma_{a}\left|A_{s}^{\prime}\right|^{2} A_{a}^{\prime}\right\} \text {, } \\
& \frac{\partial A_{l}}{\partial z}+\frac{1}{v_{l}^{2}} \frac{\partial A_{l}}{\partial t}=i \frac{2 \pi \omega_{l}}{c n_{l} \cos \left(\theta_{l}^{z}\right)}\left\{\frac{4 \pi q_{p}^{2}\left(\chi_{11} \chi_{p 2}^{*}+\chi_{12} \chi_{p 1}\right)}{\left(W^{2}-q_{p}^{2} \varepsilon_{p}^{\infty}\right)} A_{a}^{\prime} A_{s}^{\prime} A_{l}^{*}\right. \\
& \left.+\frac{4 \pi q_{p}^{2} \chi_{12} \chi_{p 2}}{\left(W^{2}-q_{p}^{2} \varepsilon_{p}^{\infty}\right)}\left|A_{a}^{\prime}\right|^{2} A_{1}+\gamma_{11}\left|A_{s}^{\prime}\right|^{2} A_{1}+\gamma_{l}\left|A_{1}\right|^{2} A_{1}\right\} \text {, }
\end{aligned}
$$




$$
\begin{aligned}
& \frac{\partial A_{s}^{\prime}}{\partial z}+\frac{1}{v_{s}^{2}} \frac{\partial A_{s}^{\prime}}{\partial t} \\
& =i \frac{2 \pi \omega_{s}}{c n_{s} \cos \left(\theta_{s}^{z}\right)}\left\{\frac{4 \pi q_{p}^{2} \chi_{s} \chi_{p 2}}{\left(W^{2}-q_{p}^{2} \varepsilon_{p}^{\infty}\right)} A_{1}^{2} A_{a}^{\prime *}+\gamma_{s 1}\left|A_{1}\right|^{2} A_{s}^{\prime}+\gamma_{s}\left|A_{s}^{\prime}\right|^{2} A_{s}^{\prime}\right\} .
\end{aligned}
$$

\section{Asymptotic Solutions in a Form of Simultaneously Propagating Waves at Frequencies $\omega_{a, l, s}$}

Since we will conduct the numerical analysis of the system (20)-(22) we bring it to unitless form first. To do that we multiply both the left and right part of each equation by the factor $z_{0} / A_{0} \quad\left(A_{0}\right.$ and $\tau_{0}$ are the peak amplitude and characteristic duration of the pump, $\left.z_{0}=c \tau_{0}\right)$. After that, the system (20)-(22) can be reduced to

$$
\begin{gathered}
\frac{\partial \tilde{A}_{a}^{\prime}}{\partial \tilde{z}}+\frac{1}{\tilde{v}_{a}^{2}} \frac{\partial \tilde{A}_{a}^{\prime}}{\partial \tilde{t}}=i\left\{C_{a 1} \tilde{A}_{l}^{2} \tilde{A}_{s}^{\prime *}+C_{a 2}\left|\tilde{A}_{l}\right|^{2} \tilde{A}_{a}^{\prime}+C_{a 3}\left|\tilde{A}_{s}^{\prime}\right|^{2} \tilde{A}_{a}^{\prime}\right\}, \\
\frac{\partial \tilde{A}_{l}}{\partial \tilde{z}}+\frac{1}{\tilde{v}_{l}^{z}} \frac{\partial \tilde{A}_{l}}{\partial \tilde{t}}=i\left\{C_{l 1} \tilde{A}_{a}^{\prime} \tilde{A}_{s}^{\prime} \tilde{A}_{l}^{*}+C_{l 2}\left|\tilde{A}_{s}^{\prime}\right|^{2} \tilde{A}_{l}+C_{l 3}\left|\tilde{A}_{l}\right|^{2} \tilde{A}_{l}+C_{l 4}\left|\tilde{A}_{a}^{\prime}\right|^{2} \tilde{A}_{l}\right\}, \\
\frac{\partial \tilde{A}_{s}^{\prime}}{\partial \tilde{z}}+\frac{1}{\tilde{v}_{s}^{z}} \frac{\partial \tilde{A}_{s}^{\prime}}{\partial \tilde{t}}=i\left\{C_{s 1} \tilde{A}_{l}^{2} \tilde{A}_{a}^{\prime *}+C_{s 2}\left|\tilde{A}_{l}\right|^{2} \tilde{A}_{s}^{\prime}+C_{s 3}\left|\tilde{A}_{s}^{\prime}\right|^{2} \tilde{A}_{s}^{\prime}\right\},
\end{gathered}
$$

where $\tilde{A}_{a, s}^{\prime} \equiv \frac{A_{a, s}^{\prime}}{A_{0}}, \quad \tilde{A}_{1} \equiv \frac{A_{1}}{A_{0}}, \tilde{t} \equiv \frac{t}{\tau_{0}}, C_{a 1} \equiv \frac{2 \pi \omega_{a} z_{0}}{c n_{a} \cos \left(\theta_{a}^{2}\right)} \frac{4 \pi q_{p}^{2} \chi_{a} \chi_{p 1}^{*} A_{0}^{2}}{\left(W^{2}-q_{p}^{2} \varepsilon_{p}^{\infty}\right)}$,

$$
\begin{gathered}
C_{a 2} \equiv \frac{2 \pi \omega_{a} z_{0}}{c n_{a} \cos \left(\theta_{a}^{z}\right)} \gamma_{a 1} A_{0}^{2}, C_{a 3} \equiv \frac{2 \pi \omega_{0} z_{0}}{c n_{a} \cos \left(\theta_{a}^{z}\right)} \gamma_{a} A_{0}^{2}, C_{l 2} \equiv \frac{2 \pi \omega_{l} z_{0}}{c n_{l} \cos \left(\theta_{l}^{z}\right)} \gamma_{l 1} A_{0}^{2}, \\
C_{l 3} \equiv \frac{2 \pi \omega_{l} z_{0}}{c n_{l} \cos \left(\theta_{l}^{z}\right)} \gamma_{l} A_{0}^{2}, C_{l 1} \equiv \frac{2 \pi \omega_{l} z_{0}}{c n_{l} \cos \left(\theta_{l}^{z}\right)} \frac{4 \pi q_{p}^{2}\left(\chi_{l 1} \chi_{p 2}^{*}+\chi_{l 2} \chi_{p 1}\right) A_{0}^{2}}{\left(W^{2}-q_{p}^{2} \varepsilon_{p}^{\infty}\right)}, \\
C_{l 4} \equiv \frac{2 \pi \omega_{l} z_{0}}{c n_{l} \cos \left(\theta_{l}^{z}\right)} \frac{4 \pi q_{p}^{2} \chi_{l 2} \chi_{p 2} A_{0}^{2}}{\left(W^{2}-q_{p}^{2} \varepsilon_{p}^{\infty}\right)}, C_{s 1} \equiv \frac{2 \pi \omega_{s} z_{0}}{c n_{s} \cos \left(\theta_{s}^{z}\right)} \frac{4 \pi q_{p}^{2} \chi_{s} \chi_{p 2} A_{0}^{2}}{\left(W^{2}-q_{p}^{2} \varepsilon_{p}^{\infty}\right)}, \\
C_{s 2} \equiv \frac{2 \pi \omega_{s} z_{0}}{c n_{s} \cos \left(\theta_{s}^{2}\right)} \gamma_{s 1} A_{0}^{2}, \quad C_{s 3} \equiv \frac{2 \pi \omega_{s} z_{0}}{c n_{s} \cos \left(\theta_{s}^{z}\right)} \gamma_{s} A_{0}^{2} .
\end{gathered}
$$

We are looking for stationary solutions as

$$
\tilde{A}_{a, s}^{\prime}(\tilde{z}, \tilde{t}) \equiv B_{a, s}(\tilde{\xi}) \mathrm{e}^{i \Phi_{a, s}(\tilde{\xi})} \text { and } \tilde{A}_{l}(\tilde{z}, \tilde{t}) \equiv B_{l}(\tilde{\xi}) \mathrm{e}^{i \Phi_{l}(\tilde{\xi})},
$$

where $\tilde{\xi} \equiv \tilde{t}-\tilde{z} / \tilde{v}^{z} ; \tilde{v}^{z}$ is the velocity of simultaneously propagating waves at the frequencies $\omega_{a, l, s} ; B_{a, l, s}$ and $\Phi_{a, l, s}$ are the real amplitudes and phases of the waves, respectively. Such a standard procedure of presenting the complex amplitudes of waves in terms of real and imaginary parts results in duplication of the system of (23)-(25):

$$
\frac{\mathrm{d} B_{a}}{\mathrm{~d} \tilde{\xi}}=-\kappa_{a} C_{a 1} B_{l}^{2} B_{s} \sin (\Phi),
$$




$$
\begin{gathered}
\frac{\mathrm{d} \Phi_{a}}{\mathrm{~d} \tilde{\xi}}=\kappa_{a}\left\{C_{a 1} \frac{B_{l}^{2} B_{s}}{B_{a}} \cos (\Phi)+C_{a 2} B_{l}^{2}+C_{a 3} B_{s}^{2}\right\}, \\
\frac{\mathrm{d} B_{l}}{\mathrm{~d} \tilde{\xi}}=\kappa_{l} C_{l 1} B_{a} B_{s} B_{l} \sin (\Phi), \\
\frac{\mathrm{d} \Phi_{l}}{\mathrm{~d} \tilde{\xi}}=\kappa_{l}\left\{C_{l 1} B_{a} B_{s} \cos (\Phi)+C_{l 2} B_{s}^{2}+C_{l 3} B_{l}^{2}+C_{l 4} B_{a}^{2}\right\}, \\
\frac{\mathrm{d} B_{s}}{\mathrm{~d} \tilde{\xi}}=-\kappa_{s} C_{s 1} B_{l}^{2} B_{a} \sin (\Phi), \\
\frac{\mathrm{d} \Phi_{s}}{\mathrm{~d} \tilde{\xi}}=\kappa_{s}\left\{C_{s 1} \frac{B_{l}^{2} B_{a}}{B_{s}} \cos (\Phi)+C_{s 2} B_{l}^{2}+C_{s 3} B_{s}^{2}\right\},
\end{gathered}
$$

where $\kappa_{a . s . l} \equiv v_{a, s, l}^{z} v^{z} /\left(v^{z}-v_{a, s, l}^{z}\right), \quad \Phi \equiv 2 \Phi_{l}-\Phi_{s}-\Phi_{a}$.

If we introduce the amplitude of simultaneously propagated waves as

$$
Q \equiv-\frac{B_{a}^{2}}{\kappa_{a} C_{a 1}}=-\frac{B_{s}^{2}}{\kappa_{s} C_{s 1}}=\frac{B_{l}^{2}}{\kappa_{l} C_{l 1}}
$$

we could reduce the system above to

$$
\begin{gathered}
\frac{\mathrm{d} Q}{\mathrm{~d} \tilde{\xi}}=\alpha Q^{2} \sin (\Phi), \\
\frac{\mathrm{d} \Phi}{\mathrm{d} \tilde{\xi}}=2 \alpha Q \cos (\Phi)+\beta Q,
\end{gathered}
$$

where

$$
\begin{aligned}
& \lambda_{a}^{2}=-\kappa_{a} C_{a 1}, \lambda_{l}^{2}=\kappa_{l} C_{l 1}, \lambda_{s}^{2}=-\kappa_{s} C_{s 1}, \alpha \equiv 2 \lambda_{a} \lambda_{s} \lambda_{l}^{2}, \\
& \beta \equiv 2 \kappa_{l}\left(C_{l 2} \lambda_{s}^{2}+C_{l 3} \lambda_{l}^{2}+C_{l 4} \lambda_{a}^{2}\right)-\kappa_{s}\left(C_{s 2} \lambda_{l}^{2}+C_{s 3} \lambda_{s}^{2}\right)-\kappa_{a}\left(C_{a 2} \lambda_{l}^{2}+C_{a 3} \lambda_{s}^{2}\right) .
\end{aligned}
$$

The system (34)-(35) can be further simplified as follows

$$
\begin{gathered}
\frac{\mathrm{d} Q}{\mathrm{~d} x}=Q^{2} \sin (\Phi), \\
\frac{\mathrm{d} \Phi}{\mathrm{d} x}=Q(2 \cos (\Phi)+\tilde{\beta}),
\end{gathered}
$$

where $x \equiv \tilde{\xi} \alpha, \quad \tilde{\beta}=\frac{\beta}{\alpha}$.

We can reduce the number of equations by using the integral of motion

$$
Q=\frac{1}{\sqrt{2 \cos (\Phi)+\tilde{\beta}}}
$$

where $Q>0, \tilde{\beta}>2$.

If we express the phase $\Phi$ as the function of $Q$ in (37) we get

$$
\int \frac{\mathrm{d} Q}{\sqrt{\left(4-\tilde{\beta}^{2}\right) Q^{4}+2 \tilde{\beta} Q^{2}-1}}=\frac{1}{2} x
$$


The integral on the left can be found as follows:

$$
\begin{aligned}
& \int \frac{\mathrm{d} Q}{\sqrt{\left(4-\tilde{\beta}^{2}\right) Q^{4}+2 \tilde{\beta} Q^{2}-1}}=-\left(i \sqrt{\left(1-(\tilde{\beta}-2) Q^{2}\right)\left(1-(\tilde{\beta}+2) Q^{2}\right)}\right. \\
& \left.\times F\left(i \sinh ^{-1}\left(\sqrt{(2-\tilde{\beta}) Q} \mid \frac{\tilde{\beta}-2}{\tilde{\beta}+2}\right)\right)\right) / \sqrt{(2-\tilde{\beta})\left(\left(4-\tilde{\beta}^{2}\right) Q^{4}+2 \tilde{\beta} Q^{2}-1\right)},
\end{aligned}
$$

where $F(\tilde{x} \mid m)$ is the elliptic integral of the first kind with the parameter $m=k^{2}, \sinh ^{-1}(\tilde{x})$ is the inverse hyperbolic sine function.

In Figure 1 it is shown that the solution of (37) and (38) exists in the form of pulses. The duration of those pulses can be easily evaluated as follows: first, we assume, that $C_{a 1} \approx C_{s 1} \approx C_{l 1} \approx C$ (in the next topic it is shown that $g \approx C$ where $g$ is the gain factor of Raman scattering) so that the coefficient $\alpha=\lambda_{a} \lambda_{l}^{2} \lambda_{s} \approx C^{2} \approx g^{2}$. The typical values of the gain factor in crystals are of order $10^{-3} \mathrm{~cm} / \mathrm{MW}$ [24]. Hence, if we consider the pump of the intensity of $10^{2}$ $10^{3} \mathrm{MW}$ and $\mathrm{z}=1 \mathrm{~cm}$, then $g \approx 1$.

\section{Gain Factor $g$}

To show that the system of Equations (5)-(8) is consistent with experimental results for CARS by polaritons we consider the stationary solutions of the coupled wave equations in the constant pump approximation. The system for Stokes and anti-Stokes (20), (22) under the above suggestions can be expressed as

$$
\begin{gathered}
\frac{\partial \tilde{A}_{s}}{\partial \tilde{Z}}=i C_{s 1} \tilde{A}_{a}^{*} e^{i \Delta k^{z} z} \\
\frac{\partial \tilde{A}_{a}^{*}}{\partial \tilde{z}}=-i C_{a 1} \tilde{A}_{s} \mathrm{e}^{-i \Delta k^{z} z}
\end{gathered}
$$

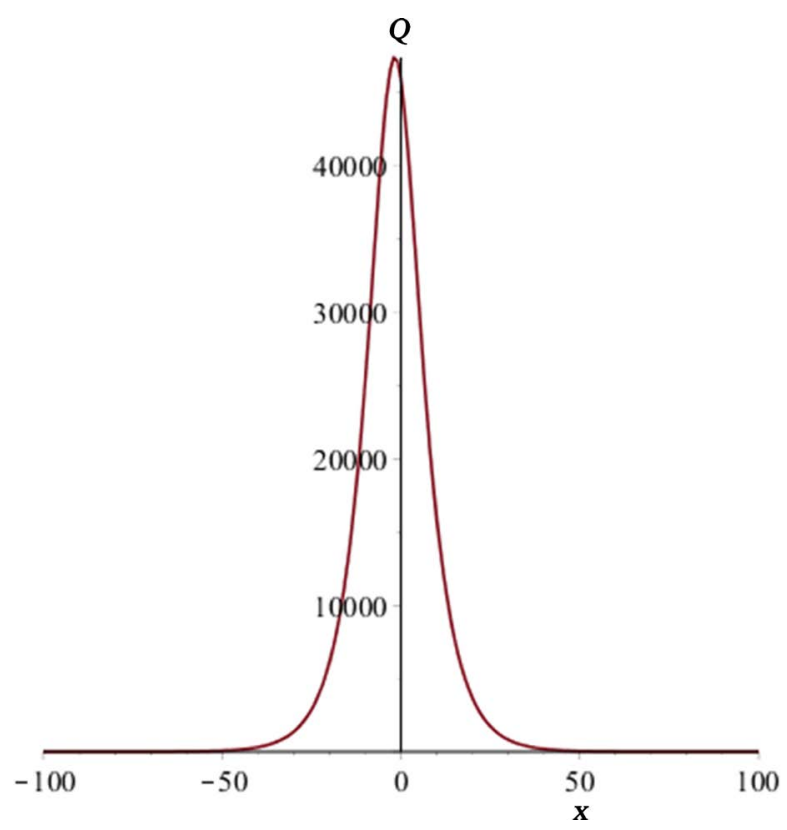

Figure 1. $Q$ versus $x$. 
After introducing new variables

$$
F_{s} \equiv \tilde{A}_{s} \mathrm{e}^{-i \Delta k^{2} z / 2} \text { and } F_{a}^{*} \equiv \tilde{A}_{a}^{*} \mathrm{e}^{i \Delta k^{2} z / 2}
$$

the system of differential equations of the first order can be readily transformed to the single differential equation of the second order (for example, for $F_{s}(z)$ ) as

$$
\frac{\partial^{2} F_{s}}{\partial z^{2}}+\left(\frac{\Delta k^{z}}{2}\right)^{2} F_{s}=C_{a 1} C_{s 1} F_{s} \text {. }
$$

We solve this equation by adopting a trial solution for $F_{s}$ in the form

$$
F_{s}(z)=F_{s}(0) \mathrm{e}^{g z}
$$

where $g$ represents a gain factor. Then we substitute (46) into (45) to obtain the approximate value for $g$ as

$$
g \approx\left(C_{a 1} C_{s 1}\right)^{\frac{1}{2}} \approx C
$$

(here we assumed that the pump was strong enough to provide $C_{a 1} C_{s 1} \gg\left(\frac{\Delta k^{z}}{2}\right)^{2}$ ).

Finally, the expression for $g$ can be reduced to

$$
\begin{gathered}
g \approx C \approx 8 \pi^{2} \omega z_{0} \chi^{2} A_{0}^{2} /(c n) \\
\left.g \approx\left(C_{a 1} C_{s 1}\right)^{\frac{1}{2}}=\left(\frac{2 \pi \omega_{a} z_{0}}{c n_{a} \cos \left(\theta_{a}^{2}\right)} \frac{4 \pi q_{p}^{2} \chi_{a} \chi_{p 1}^{*} A_{0}^{2}}{\left(W^{2}-q_{p}^{2} \varepsilon_{p}^{\infty}\right)} \frac{2 \pi \omega_{s} z_{0}}{c n_{s} \cos \left(\theta_{s}^{2}\right)} \frac{4 \pi q_{p}^{2} \chi_{s} \chi_{p 2} A_{0}^{2}}{\left(W^{2}-q_{p}^{2} \varepsilon_{p}^{\infty}\right)}\right)^{1 / 2}\right) . \\
\approx\left(8 \pi^{2} \omega z_{0} /\left(\varepsilon^{\prime} n c\right) \chi^{2} A_{0}^{2}\right) \approx 8 \pi^{2} \omega z_{0} \chi^{2} A_{0}^{2} /(c n)
\end{gathered}
$$

As the experimental data for this gain, we used the following [25]: pulse width of the pulsed $\mathrm{Ar}^{+}$laser $\simeq 30 \mathrm{ps}$, the peak output power $\approx 2.5 \mathrm{~kW}$, the wavelength was $514.5 \mathrm{~nm}$, the cross-section $\approx 10^{-18} \mathrm{~cm}^{-2}, \gamma_{f} \approx 10 \mathrm{~cm}^{-1}$, and $\chi \approx 10^{-8} \mathrm{esu}$. In [26] the nonlinear medium was zinc blende $\mathrm{ZnS}$, in which the polariton frequencies were in the rage $200-400 \mathrm{~cm}^{-1}$. Both the experimental results for the gain factor in [26] and calculations based on (48) have resulted in $g \approx 1$.

\section{Conclusion}

In this paper, we have found the system of differential equations that model the process of coherent anti-Stokes Raman scattering by polaritons in crystals. We have also found the asymptotic solutions of that system that correspond to the simultaneous propagation of all waves participating in the process of Raman scattering. And, lastly, we showed that the value of such an important feature as the gain factor resulted from that system is consistent with the experimental results.

\section{Conflicts of Interest}

The authors declare no conflicts of interest regarding the publication of this paper.

\section{References}

[1] Maker, P.D. and Terhune R.W. (1965) Study of Optical Effects Due to an Induced 
Polarization of Third Order in the Electric Field Strength. Physical Review, 137, A801-A818. https://doi.org/10.1103/PhysRev.137.A801

[2] Levenson, M.D. (1972) Interference of Resonant and Nonresonant Three-Wave Mixing in a Diamond. Physical Review, 86, 3962-3965.

https://doi.org/10.1103/PhysRevB.6.3962

[3] Levenson, M.D. (1974) Feasibility of Measuring the Nonlinear Index of Refraction by Third-Order Frequency Mixing. IEEE Journal of Quantum Electronics, QE-10, 110-115.

[4] Wynne, J.J. (1972) Nonlinear Optical Spectroscopy of $\chi^{(3)}$ in $\mathrm{LiNbO}_{3}$. Physical Review Letters, 29, 650-653. https://doi.org/10.1103/PhysRevLett.29.650

[5] Tolles, W.M., Nibler, J.W., McDonald, J.R. and Harvey, A.B. (1977) A Review of the Theory and Application of Coherent Anti-Stokes Raman Spectroscopy. Applied Spectroscopy, 31, 253. https://doi.org/10.1366/000370277774463625

[6] Zheltikov, A.M. (2000) Coherent Anti-Stokes Raman Scattering: From Proof-of-the Principle Experiments to Femtosecond CARS and Higher-Order Wave-Mixing Generalizations. Journal of Raman Spectroscopy 31, 653-667.

https://doi.org/10.1002/1097-4555(200008/09)31:8/9\%3C653::AID-JRS597\%3E3.0.C $\mathrm{O} ; 2-\mathrm{W}$

[7] Shen, Y., Voronine, D.V. Sokolov, A.V., and. Scully, M.O. (2015) A Versatile Setup Using Femtosecond Adaptive Spectroscopic Techniques for Coherent Anti-Stokes Raman Scattering. Review of Scientific Instruments, 86, Article ID: 083107. https://doi.org/10.1063/1.4929380

[8] Ariunbold, G.O. and Altangerel, N. (2016) Coherent Anti-Stokes Raman Spectroscopy: Understanding the Essentials. Journal of Raman Spectroscopy, 3, 6-17. https://doi.org/10.1515/coph-2016-0002

[9] Duncan, M., Reintjes, J. and Manuccia, T.J. (1982) Scanning Coherent Anti-Stokes Raman Microscope. Optics Letters, 7, 350-352. https://doi.org/10.1364/OL.7.000350

[10] Potcoava, M.C., Futia, G.L., Aughenbaugh, J., Schlaepfer, I.R. and Gibson, E.A. (2014) Raman and Coherent Anti-Stokes Raman Scattering Microscopy Studies of Changes in Lipid Content and Composition in Hormone-Treated Breast and Prostate Cancer Cells. Journal of Biomedical Optics, 19, Article ID: 111605. https://doi.org/10.1117/1.JBO.19.11.111605

[11] Le, T.T., Huff, T.B. and Cheng, J.X. (2009) Coherent Anti-Stokes Raman Scattering Imaging of Lipids in Cancer Metastasis. BMC Cancer, 9, Article No. 42. https://doi.org/10.1186/1471-2407-9-42

[12] Begley, R.F., Harvey, A.B. and Byer, R.L. (1974) Coherent Anti-Stokes Raman Spectroscopy. Applied Physics Letters, 25, 387-390. https://doi.org/10.1063/1.1655519

[13] Harris, I.E. and McIlwain, M.E. (1981) Coherent Anti-Stokes Raman Spectroscopy in Propellant Flames. In: Capellos, C. and Walker, R.F., Eds., Fast Reactions in Energetic Systems, Springer, Dordrecht, 473-484.

https://doi.org/10.1007/978-94-009-8511-7_23

[14] Hall, R.J. and Eckbreth, A.C. (1982) Coherent Anti-Stokes Raman Spectroscopy: Applications to Combustion Diagnostics. In: Erf, R.K., Ed. Laser Applications, Academic Press, New York.

[15] Leonhardt, R., Holzaptel, W., Zinth, W. and Kaizer, W. (1987) Terahertz Quantum Beats in Molecular Liquids. Chemical Physics Letters, 133, 373-377. https://doi.org/10.1016/0009-2614(87)87085-9

[16] Mukamel, S. (1990) Femtosecond Optical Spectroscopy: A Direct Look at Elemen- 
tary Chemical Events. Annual Review of Physical Chemistry, 41, 647-681. https://doi.org/10.1146/annurev.pc.41.100190.003243

[17] Nelson, K.A. and Ippen, E.P. (1989) Femtosecond Coherent Spectroscopy. In: Prigogine, I. and Rice, S.A., Eds., Advances in Chemical Physics, John Wiley \& Sons, Inc., Hoboken, 1-35. https://doi.org/10.1002/9780470141243.ch1

[18] Dyakov, Y.S., Krikunov, S.A., Magnitskii, C.A., Nikitin, S.Y. and Tunkin, V.G. Time-Domain Coherent Anti-Stokes Raman Spectroscopy for Hydrogen Gas in the Dicke Narrowing Region. Zh. Exp. Teor. Fiz, 84, 2013-2025.

[19] Feshchenko, G. and Feshchenko, V. (2015) Computer Simulation of Transition Regimes of Solitons in Stimulated Raman Scattering with Excitation of Polar Optical Phonons. American Journal of Computational Mathematics, 5, 336-344. https://doi.org/10.4236/ajcm.2015.53031

[20] Feshchenko, V. and Feshchenko, G. (2018) Nonstationary Stimulated Raman Scattering by Polaritons in Continuum of Dipole-Active Phonons. Journal of Applied Mathematics and Physics, 6, 405-417. https://doi.org/10.4236/jamp.2018.62038

[21] Feshchenko, V. and Feshchenko, G. (2019) Nonstationary Stimulated Raman Scattering by Polaritons in Cubic Crystals. Journal of Applied Mathematics and Physics, 7, 2122-2129. https://doi.org/10.4236/jamp.2019.79145

[22] Ahmanov S.A. and Hohlov, R.V. (1964) Problems of Nonlinear Optics. VINITI, Moscow.

[23] Strizhevskii, V.L. (1972) Theory of Stimulated Raman Scattering by Polaritons in Cubic and Uniaxial Crystals. Zh. Exp. Teor. Fiz, 35, 760-766.

[24] Boyd, R. (2019) Nonlinear Optics. 4th Edition, Elsevier, Amsterdam.

[25] Harvey, J.D., Proctor, M.J. and Steed, C.A. (1988) 30 Ps Cavity-Dumped Pulses from an Argon Laser. Applied Physics Letters, 52, 688. https://doi.org/10.1063/1.99348

[26] Cheng, Y.C., Jin, C.Q., Gao, F., Wu, X.L., Zhong, W., Li, S.H., and Chu, P.K. (2009) Raman Scattering Study of Zinc Blende and Wurtzite ZnS. Journal of Applied Physics, 106, Article ID: 123505. https://doi.org/10.1063/1.3270401 